\title{
FACTORIZATION OF K-QUASIHYPONORMAL OPERATORS
}

\author{
S. C. ARORA \\ Department of Mathematics \\ University of Delhi \\ Delhi - 110007 \\ Inti.a \\ and \\ J.K. THUKRAL \\ Department of Mathematics \\ S.R.C.C. \\ University of Delhi \\ Delhi - 110007 \\ India
}

(Received January 1, 1987 and in revised form March 17, 1989)

ABSTRACT. Let $A$ be the class of all operators $T$ on a Hilbert space $H$ such that $R\left(T \star^{k} T\right)$, the range space of $T^{k} T$, is contained in $R\left(T^{k+1}\right)$, for a positive integer $k$. It has been shown that if $T \in A$, there exists a unique operator $C_{T}$ on $H$ such that

(i) $\quad \mathrm{T} * \mathrm{k}^{\mathrm{T}}=\mathrm{T}^{*^{\mathrm{k}+1}} \mathrm{C}_{\mathrm{T}}$;

(ii) $\quad\left\|C_{T}\right\|^{2}=\inf \left\{\mu: \mu>0\right.$ and $\left.\left(T *^{k} T\right)\left(T *^{k} T\right) *<\mu T \star^{k+1} T^{k+1}\right\}$;

(i1i) $N\left(C_{T}\right)=N\left(T *^{k} T\right)$ and

(iv) $R\left(C_{T}\right) \subseteq \overline{R\left(T^{k+1}\right)}$

The main objective of this paper is to characterize k-quasihyponormal; normal, and self-adjoint operators $T$ in $A$ in terms of $C_{T}$. Throughout the paper, unless stated otherwise, $H$ will denote a complex Hilbert space and $T$ an operator on $H, i . e ., a$ bounded linear transformation from $H$ into $H$ itself. For an operator $T$, we write $R(T)$ and $N(T)$ to denote the range space and the null space of $T$.

KEY WORDS AND PHRASES. Self-adjoint, normal, unitary, quasinormal, hyponormal, quasihyponormal, k-quasihyponormal, isometry, partial lsometry, null space, range space and the projection.

1980 AMS SUBJECT CLASSIFICATION CODES. 47B20; 47B15.

\section{INTRODUCTION}

$T$ is said to be quasinormal if $T(T * T)=(T * T) T$, hyponormal if $T * T>T T *$ or equivalently $\left\|T^{*} x||<\right\| T x \|$ for each $x$ in $H, k$-quasihyponormal (Campbell and Gupta 
[1]) for a positive integer $k$ if $T \star^{k}(T \star T-T T *) T^{k}>0$ or equivalently $\left\|T * T^{k} x\right\|<\left\|T^{k+1} x\right\|$ for each $x$ in $H$.

The purpose of this paper is to consider the class A of those operators $T$ such that $R\left(T \star^{k} T\right) \subseteq R\left(T^{k}{ }^{k+1}\right)$ for a positive integer $k$. More precisely, our ain is to identify those operators $T$ in $A$ which ars $k$-quasihyponormal, normal and selfadjoint. The motivation is due to Embry [2] who considered the class of operators $T$ satisfying $R(T) \subseteq R\left(T^{*}\right)$ and Patel [5] who discussed the class of operators $T$ satisfying $R(T * T) \subseteq R\left(T *^{2}\right)$. If $T \in A$, then by Douglas' theorem [3, Theorem 1] there exists a unique operator $C_{\mathrm{T}}$ such that

(1) $\quad \mathrm{T}^{k} \mathrm{~T}=\mathrm{T}^{k+1} \mathrm{C}_{\mathrm{T}}$;

(ii) $\quad\left\|C_{T}\right\|^{2}=\inf \left\{\mu: \mu>0\right.$ and $\left(T *^{k} T\right)\left(T *^{k} T\right) *\left\langle\mu \Gamma \star^{k+1} T^{k+1}\right\}$;

(iii) $\quad \mathrm{N}\left(\mathrm{C}_{\mathrm{T}}\right)=\mathrm{N}\left(\mathrm{T} *^{k} \mathrm{~T}\right)$ : and

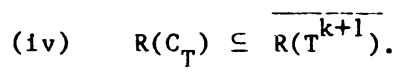

2. MAIN RESUlts.

By Douglas' theorem [3, Theorem 1], the class A contains all k-quasihyponormal operators.

THEOREM 2.1. An operator $T$ in $A$ is $k$-quasthyponormal if and only if $\left\|C_{T}\right\|<1$. PROOF. If $\left\|\mathrm{C}_{\mathrm{T}}\right\|<1,\left\|\mathrm{~T}^{\star} \mathrm{T}^{k} \mathrm{x}\right\|=\left\|\mathrm{C}_{\mathrm{T}}{ }^{*} \mathrm{~T}^{\mathrm{k}+\mathrm{l}} \mathrm{x}\right\|<\left\|\mathrm{T}^{k+1} \mathrm{x}\right\|$

for all $x$ in $H$ and hence $T$ is $k$-quasihyponormal.

Conversely, assume that $T$ is $k$-quasihyponormal. Since

$$
\left\|C_{T}^{*} T^{k+1} x\right\|=\left\|T^{\star} T^{k} x\right\|<\left\|T^{k+1} x\right\|
$$

for all $x$ in $H,\left\|C_{T}^{* y}\right\|<\|y\|$ for all $y$ in $\overline{R\left(T^{k+1}\right)}$. Also since $R\left(C_{T}\right) \subseteq \overline{R\left(T^{k+1}\right)}$, i.e. $\overline{R\left(T^{k+1}\right)} \subseteq N\left(C_{T}^{*}\right)$, we have $C^{*} T^{x}=0$ for all $x$ in $\overline{R\left(T^{k+1}\right)}{ }^{\perp}$. Thus for each $x$ in $\mathrm{H},\left\|\mathrm{C}_{\mathrm{T}}^{\star} \mathrm{x}\right\|<\|\mathrm{x}\|$ and consequently $\left\|\mathrm{C}_{\mathrm{T}}\right\|=\left\|\mathrm{C}_{\mathrm{T}}^{\star}\right\|<1$.

To prove our next result, we need the following lemma.

LEMMA 2.1. Let $T$ be a quasinormal operator. Then for any positive integer $k$
(a) $T * T^{k}=T^{k-1} T * T$
(b) $\left\|(T * T)^{k / 2} x\right\|=\left\|T^{k} x\right\|$ for all vectors $x$ in $H$
(c) $N\left(T *^{k} T\right) \subseteq N\left(T *^{k}\right)$.

PROOF. (a) We prove $i t$ by induction on $k$. For $k=1$, trivial. For $k=2$, again it holds since $T$ is quasinormal. Now assume that the result is true for any positive integer $m>2$. Then $T * T^{m+1}=\left(T * T^{m}\right) T=\left(T^{m-1} T * T\right) T=T^{m-1}(T * T) T=T^{m-1} T T * T=T^{m} T * T$. Hence by induction the result follows. (b) It is an immediate consequence of the fact that if $T$ is quasinormal, then $\left(T^{*} T\right)^{k}=T^{* k} T^{k}$ for any positive integer $k$. (c) 
Let $x \in N\left(T \star^{k} T\right)$. Then $T \star^{k} T x=0$, 1.e., $T \star^{* T} T \star^{k-1} x=0$ by (a). Thus $T \star^{k-1} x \in N(T \star T)=$ $N(T)$. But $N(T) \subseteq N(T *)$ since $T$ is quasinormal. Therefore $T^{*^{k}} x=0$, i.e., $x \in N\left(T^{*}\right)$.

By using the lemma we obtain the following

THEOREM 2.2. Let $T \in A$ be a quasinormal operator. Then $C_{T}$ is a quasinormal

partial isometry with $R\left(C_{T}\right)=\overline{R\left(T^{k+1}\right)}$.

PROOF. We have $\left\|C_{T}^{\star} T^{k+1} x\right\|=\left\|T^{*} T^{k} x\right\|=\left\|T^{k-1} T * T x\right\|=$ $\left\|(T * T)^{k-1 / 2} \frac{T * T x}{k+1}\right\|=\left\|(T * T)^{k+1 / 2} x\right\|=\left\|T^{k+1} x\right\|$ for any $x$ in $H$. Thus $C_{T}^{*}$ is an isometry on $\overline{R\left(T^{k+1}\right) \text {. But } R\left(T^{k+1}\right) \supseteq R\left(C_{T}\right)=N\left(C_{T}^{\star}\right)}{ }^{\perp}$. Therefore $C_{T}^{\star}$ hence $C_{T}$ is a partial isometry. Further, since the initial space of a partial isometry $S$ equals the set of all those vectors $x$ satisfying $\|S x\|=\|x\|[4, p .63]$ and since $C_{T}^{\star}$ is an isometry on $\overline{R\left(T^{k+1}\right)}$, therefore $\overline{R\left(T^{k+1}\right) \subseteq N\left(C_{T}^{*}\right)}{ }^{\perp}$, the initial space of $C_{T}^{*}$. Hence $\overline{R\left(T^{k+1}\right)}=N\left(C_{T}^{*}\right)^{\perp}=\overline{R\left(C_{T}\right)}=R\left(C_{T}\right)$ as $R\left(C_{T}\right)$ is closed.

We now prove that $C_{T}$ is quasinormal. By making use of Lemma 2.1 again, we see that $N\left(C_{T}\right)=N\left(T^{k} T\right) \subseteq N\left(T^{k}\right) \subseteq N\left(T^{k}{ }^{k+1}\right)=N\left(C_{T}^{*}\right)$ since $R\left(C_{T}\right)=\overline{R\left(T^{k+1}\right)}$. From this it follows that $N\left(C_{T}\right)^{\perp}$ reduces $C_{T}$ and since $C_{T}$ is a partial isometry, $C_{T}$ is of the form $A \oplus 0$, where $A$ is an 1sometry. This gives that $C_{T}$ commutes with $C^{*}{ }_{T} C_{T}$ and hence $C_{T}$ Is quasinormal.

LEMMA 2.2. Let $T \in A$ be such that $R\left(C_{T}\right)=\overline{R(T)}$. Then $N\left(T *^{k} T\right)=N(T)$. PROOF. Since $R\left(C_{T}\right) \subseteq \overline{R\left(T^{k+1}\right)} \subseteq \ldots \subseteq \overline{R(T)}$ and, by hypothesis, $R\left(C_{T}\right)=\overline{R(T)}$, we have $R\left(C_{T}\right)=\overline{R\left(T^{k+1}\right)}=\ldots=\overline{R(T)}$. Thus $N(T *)=N\left(T \star^{2}\right)=\ldots=N\left(T \star^{k}\right)=N\left(T \star^{k+1}\right)$. Now, if $x \in N\left(T *^{k} T\right)$, then $T *^{k} T x=0$, 1.e. $T x \in N\left(T *^{k}\right)=N(T *)$. That means $T * T X=0$ or $x \varepsilon N(T * T)=N(T)$. This completes the proof.

Our next result gives a characterization of normal operators in $A$. THEOREM 2.3. An operator $T$ in $A$ is normal if and only if $C_{T}$ is a normal partial isometry with $R\left(C_{T}\right)=\overline{R(T)}$.

PROOF. Let $\mathrm{T}$ be normal. Then by Theorem 2.2, $\mathrm{C}_{\mathrm{T}}$ is a partial 1sometry with $R\left(C_{T}\right)=\overline{R\left(T^{k+1}\right)}$ and hence $R\left(C_{T}\right)=N\left(T *^{k+1}\right)^{\perp}=N(T *)^{\perp}=\overline{R(T)}$. Thus by Lemma 2.2, $N\left(C_{T}\right)=N\left(T^{*}{ }^{k}\right)=N(T)$. Therefore $R\left(C_{T}\right)=\overline{R(T)}=N\left(T^{*}\right)^{\perp}=N(T)^{\perp}=N\left(C_{T}\right)^{\perp}=R\left(C * T^{*}\right)$. Since $C_{T}^{*} C_{T}$ is the projection on $R\left(C_{T}^{*}\right)$ and $C_{T} C_{T}^{*}$ is the projection on $R\left(C_{T}\right)$, we conclude that $\mathrm{C}_{\mathrm{T}} \mathrm{C}_{\mathrm{T}}^{*}=\mathrm{C}_{\mathrm{T}}^{*} \mathrm{C}_{\mathrm{T}}$.

Assume on the other hand that $C_{T}$ is a normal partial isometry with $R\left(C_{T}\right)=R(T)$. Since $R\left(C_{T}\right) \subseteq \overline{R\left(T^{k+1}\right)} \subseteq \overline{R\left(T^{k}\right)} \subseteq \ldots \subseteq \overline{R(T)}, \quad$ we have $\left.R\left(C_{T}\right)=\overline{R\left(T^{k+1}\right.}\right)$ $=\overline{R\left(T^{k}\right)}=\ldots=\overline{R(T)}$ and consequent $1 y N(T *)=N\left(T^{*}\right)=\ldots=N\left(C T^{2}\right)=N\left(C_{T}\right)=N\left(T \star^{k} T\right)=$ 
$N(T)$ by Lemma 2.2. Thus $\|T * x\|=\|T x\|$ for each $x$ in $R\left(T^{k}\right)$. Further since $C *_{T}$ is a partial isometry on $R\left(C_{T}\right)=R\left(T^{k+1}\right)$, we have $\left\|T * T^{k} x\right\|=\left\|C * T^{k+1} x\right\|=\left\|T^{k+1} x\right\|$ for each $x$ in $H$. Thus $\|\mathrm{T} * \mathrm{y}\|=\|\mathrm{Ty}\|$ for each $y \ln \overline{\mathrm{R}}\left(\mathrm{T}^{\mathrm{k}}\right)$. Hence $\left\|\mathrm{T} \star_{\mathrm{x}}\right\|=\|\mathrm{Tx}\|$ for each $x$ in $H$, i.e., $T$ is normal.

COROLlary 2.1. Let $T \in A$. Then $T$ is normal and one-to-one if and only if $\mathrm{C}_{\mathrm{r}}$ is a unitary operator with $R\left(C_{T}\right)=\overline{R(T)}$.

PROOF. Suppose $T$ is normal and one-to-one. Then by Theorem 2.3, $C_{T}$ is a normal partial isometry with $\left.R\left(C_{T}\right)=\overline{R(T}\right)$. Since $N\left(C_{T}\right)=N(T)=\{0\}$, we have $N\left(C_{T}\right)^{\perp}=H$ and thus $C_{T}$ is an isometry and consequently $C_{T}$ is a unitary operator.

Conversely, if $C_{T}$ is a unitary operator with $R\left(C_{T}\right)=\overline{R(T)}, T$ is normal by Theorem 2.3. Also by Lemma $2.2, N(T)=N\left(T *^{k} T\right)=N\left(C_{T}\right)=\{0\}$, therefore $T$ is one-to-one.

The next corollary characterizes self-adjoint operators in $A$.

COROLLARY 2.2. Let $T \in A$. $T$ is self-adjoint if and only if $C_{T}$ is the projection on $\overline{R(T)}$.

PROOF. Suppose $T$ is self-adjoint. Then by Theorem $2.3, R\left(C_{T}\right)=\overline{R(T)}=\bar{R}\left(T^{k} \overline{1}\right)$. Since $T *^{k} T=T \star^{k+1} C_{T}$ and $T$ is self-adjoint, we have $T^{k+1}=T^{k+1} C_{T}$, i.e., $C_{T}^{\star} T^{k+1}=T^{k+1}$. This means $C_{T}^{*}=I$ on $\overline{R\left(T^{k+1}\right)}=\overline{R(T)}$. Also $C_{T}^{*}=0$ on $\overline{R(T)}{ }^{\perp}$

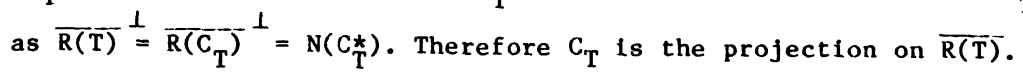

Assume now that $C_{T}$ is the projection on $\overline{R(T)}$. Then $R\left(C_{T}\right)=\overline{R(T)}$ and hence by Lemma 2.2, $N\left(C_{T}\right)=N\left(T^{*} T\right)=N(T)$. Also, as in the proof of Theorem 2.3, we have $R\left(C_{T}\right)=\overline{R\left(T^{k+1}\right)}=\ldots=\overline{R(T)}$ and thus $N(T *)=N\left(T *^{2}\right)=\ldots=N\left(C_{T}^{*}\right)=N\left(C_{T}\right)=N(T)$. Therefore $T^{\star} X=T_{X}$ for all $x$ in $R\left(T^{k}\right)$. Moreover $T^{*} T_{T}=T^{* k+1} C_{T}$, 1mplies $\mathrm{T} * \mathrm{~T}^{\mathrm{k}}=\mathrm{C}_{\mathrm{T}} \mathrm{T}^{\mathrm{k}+1}$ as $\mathrm{C}_{\mathrm{T}}$ is self-adjoint. But $\mathrm{C}_{\mathrm{T}}$ is the projection on $\overline{\mathrm{R}(\mathrm{T})}=\overline{\mathrm{R}\left(\mathrm{T}^{\mathrm{k}+\mathrm{I}}\right)}$, therefore $C_{T} T^{k+1}=T^{k+1}$, That means $T^{*} y=T y$ for all y in $\overline{R\left(T^{k}\right)}$. Thus $T^{*} x=T x$ for all $x$ in $H$ or $T$ is self-adjoint.

ACKNOWLEDGEMENT. The author gratefully acknowledges the financial support by the U.G.C. India, vide Research Grant No. F-26-1(1629)/85(SR-IV). The authors are thankful to Professor B.S. Yadav for his kind guldance and encouragement during the preparation of this paper. Thanks are also due to the referee for suggesting an improvement over the original version of the paper.

\section{REFERENCES}

1. CAMPBELL, S.L. and GUPTA, B.C., On k-Quasihyponormal Operators, Math. Japonica 23 (1978), 185-189.

2. EMBRY, M.R., Factorization of Hyponormal Operators, J. Austral. Math. Soc. 13 (1972), 323-326.

3. DOUGLAS, R.G., On Majorization, Factorization, and Range Inclusion of Operators on Hilbert space, Proc. Amer. Math. Soc. 17 (1966), 413-415.

4. HaLmoS, P.R., A Hilbert Space Problem Book, Van Nostrand, Princeton, N.J., 1967.

5. PATEL, S.M., Factorization of Quasihyponormal Operators, Publ. de 1'inst. Math. 24(38), (1978), 121-123. 


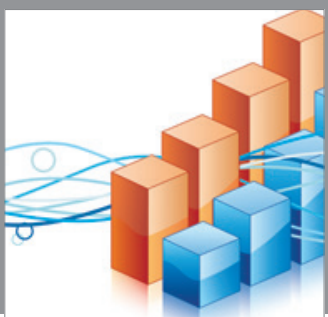

Advances in

Operations Research

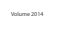

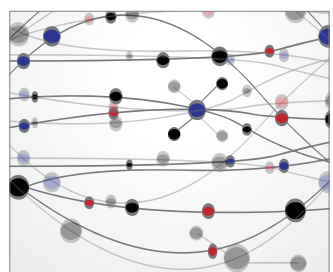

\section{The Scientific} World Journal
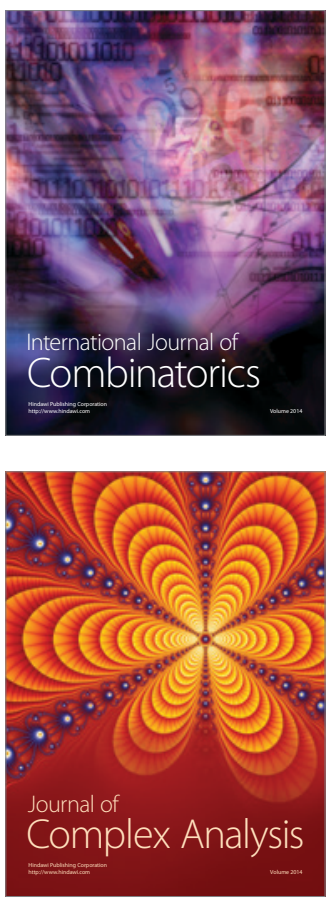

International Journal of

Mathematics and

Mathematical

Sciences
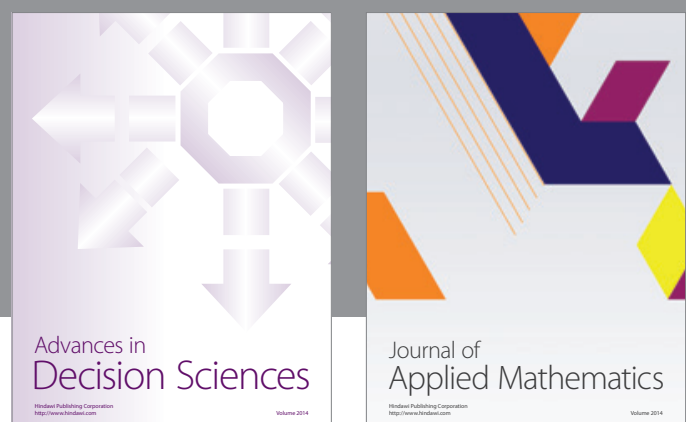

Journal of

Applied Mathematics
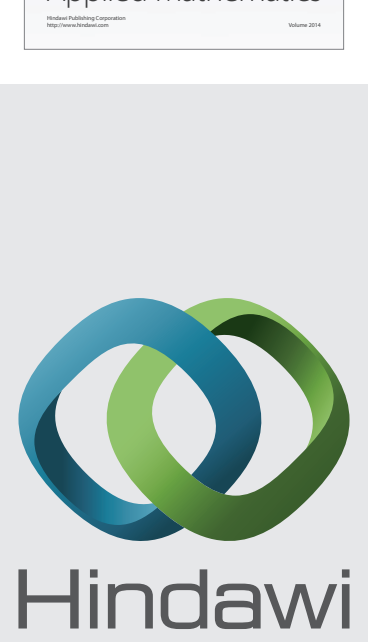

Submit your manuscripts at http://www.hindawi.com
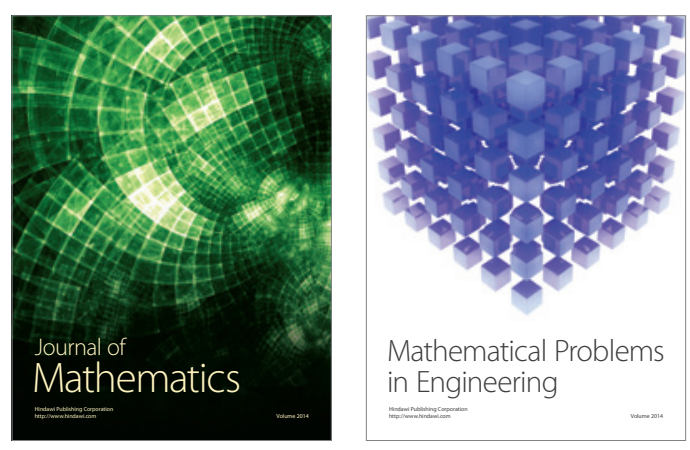

Mathematical Problems in Engineering
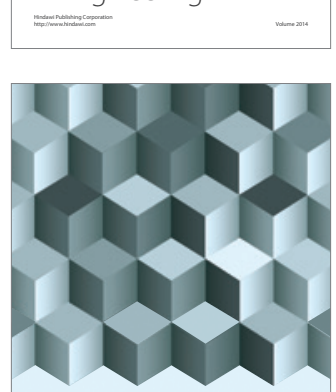

Journal of

Function Spaces
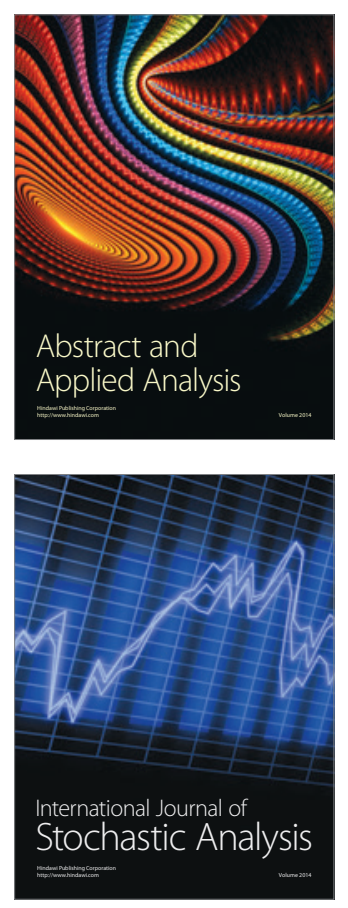

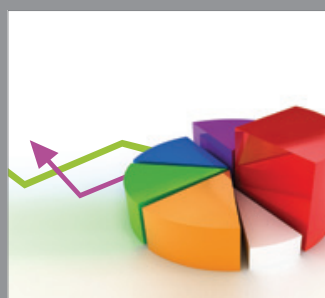

ournal of

Probability and Statistics

Promensencen
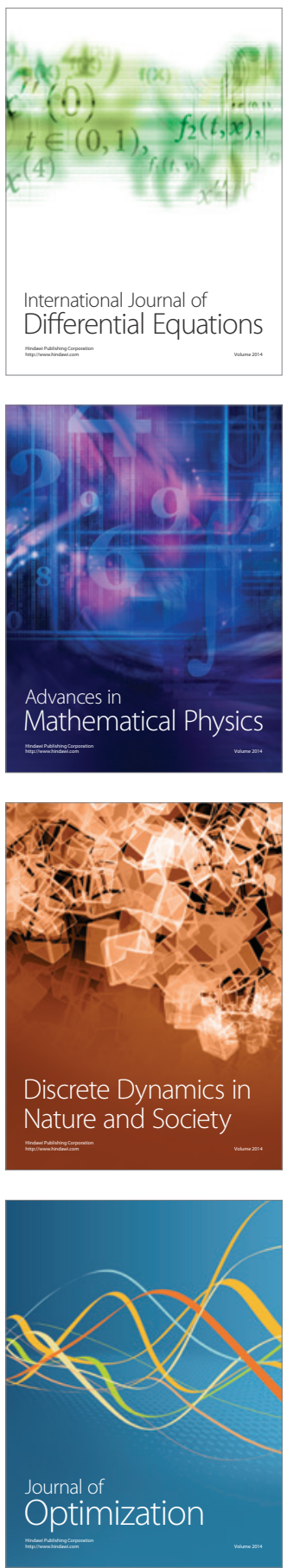